\title{
Is the Metronome-Paced Tachypnea Test (MPT) Ready for Clinical Use? Accuracy of the MPT in a Prospective and Clinical Study
}

\author{
Denise Mannée $^{\mathrm{a}} \quad$ Esther Vis $^{\mathrm{b}} \quad$ Aranka Hoekstra-Kuik $^{\mathrm{c}}$ Jan van der Maten ${ }^{\mathrm{c}}$ \\ Alex Jan van 't Hul ${ }^{a}$ Hanneke van Helvoort ${ }^{a}$

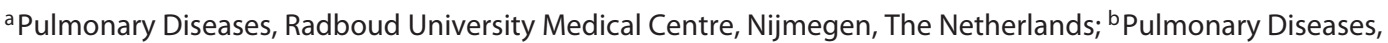 \\ Admiraal de Ruyter Ziekenhuis, Goes, The Netherlands; 'Pulmonary Diseases, Medisch Centrum Leeuwarden, \\ Leeuwarden, The Netherlands
}

\section{Keywords}

COPD · Dynamic hyperinflation · Inspiratory capacity ·

Metronome-paced tachypnea test · Diagnostic accuracy

\begin{abstract}
Background: A simple technique to measure dynamic hyperinflation $(\mathrm{DH})$ in patients with chronic obstructive pulmonary disease (COPD) is the metronome-paced tachypnea test (MPT). Earlier studies show conflicting results about the accuracy of the MPT compared to cardiopulmonary exercise testing (CPET). Objectives: The focus was to investigate the diagnostic accuracy of MPT to detect DH in a prospective and clinical study. Methods: COPD patients were included; all underwent spirometry, CPET, and MPT. DH $(\triangle \mathrm{IC})$ was calculated as the difference in \% between inspiratory capacity (IC) at the start and end of the test divided by IC at the start. A subject was identified as a hyperinflator, if $\triangle \mathrm{IC}$ (\% of ICrest) was smaller than -10.2 and $-11.1 \%$ in CPET and MPT, respectively. With these values, sensitivity and specificity were calculated. Bland-Altman plots were made of $\triangle \mathrm{IC}$ (\% of ICrest). Results: In the prospective and clinical study, 107 and 48 patients were included, respectively. Sensitivity of the MPT was $85 \%$ in both studies. The specificities were 33 and $27 \%$, re-
\end{abstract}

\begin{tabular}{ll}
\hline KARGER & $\begin{array}{l}\text { (c) } 2019 \text { The Author(s) } \\
\text { Published by S. Karger AG, Basel }\end{array}$ \\
E-Mail karger@karger.com & This article is licensed under the Creative Commons Attribution- \\
www.karger.com/res & $\begin{array}{l}\text { NonCommercial-NoDerivatives 4.0 International License (CC BY- } \\
\text { NC-ND) (http://www.karger.com/Services/OpenAccessLicense). } \\
\text { Usage and distribution for commercial purposes as well as any dis- } \\
\text { tribution of modified material requires written permission. }\end{array}$
\end{tabular}

spectively. In the prospective study, $B=+2.6 \%, L=30.6$, and $-25.6 \%$. In the clinical study, $B=+0.8 \%, L=31.0$, and $-29.1 \%$. Conclusion: MPT seems to be a good replacement for CPET in group studies. The mean amount of $\mathrm{DH}$ was not different between CPET and MPT. On an individual level, MPT cannot be used to identify hyperinflators; it should be kept in mind that MPT overdiagnoses DH. The amount of DH should not be interchanged between CPET and MPT.

\section{(C) 2019 The Author(s)}

Published by S. Karger AG, Basel

\section{Introduction}

Nowadays, cardiopulmonary exercise testing (CPET) is used clinically to quantify dynamic hyperinflation (DH). During CPET, DH is measured indirectly by serial measurement of the inspiratory capacity (IC) at the start and end of the test. However, it is a rather complex and time-consuming test. A simpler and already used technique is the metronome-paced tachypnea test (MPT). The MPT test was first used by Gelb et al. [1], in

D. Mannée and E. Vis contributed equally to this article. 
16 chronic obstructive pulmonary disease (COPD) patients. Gelb et al. [1] showed that DH with MPT was similar to DH with a CPET, if a subject breathed at twice the resting respiratory rate for $20 \mathrm{~s}$. Since, the MPT test has been used in multiple studies to investigate the response to various bronchodilators [1-4], the development of DH during exacerbations [5], the role of DH in COPD [6], and the development of DH over the course of years [7]. Despite its increasing use as a valid measurement tool, only little is known about the accuracy of the MPT. Therefore, it is unknown whether the MPT test can be used to determine $\mathrm{DH}$ in the individual patient in clinical practice.

Previous research has shown that there are conflicting results regarding the accuracy of the MPT test. Two studies exist investigating the accuracy of the MPT test based on comparison with CPET. The study by Calligaro et al. [3] describes the performance and standardization of MPT. They reported a sensitivity of $90 \%$ and a specificity of 38\%. Lahaije et al. [8] found a better accuracy, with a sensitivity and specificity of $85 \%$. Both previously published studies are limited to a small and/ or specific group of COPD patients. In the study by Calligaro et al. [3], only 24 COPD patients were included, from which 23 patients were in stage GOLD II. Lahaije et al. [8] included a larger group of COPD patients; i.e., 60 patients. However, the included patients were no good representation of an average COPD population, as the they were recruited from a pool of patients who were screened for pulmonary rehabilitation. Moreover, both studies were prospective and performed in a research setting, suggesting that MPT and CPET were performed under strict conditions and with the focus on correct execution of both tests. Therefore, the accuracy found in both studies may not be applicable to a clinical setting.

In this study, the focus was to find a conclusive answer considering the diagnostic accuracy of MPT to detect DH. In the design of this study, the limitations of both studies (Calligaro et al. [3] and Lahaije et al. [8]) were considered. This study can be divided into two parts, a prospective and retrospective clinical part. In the prospective study, a large group of COPD patients was included, and it was ensured that the group is a good representation of an average COPD population. In the clinical study, the accuracy was studied under less strict conditions. In this way, the accuracy of MPT was determined in clinical practice, where the MPT test was used as a standard lung function test in the diagnostic assessment of COPD patients.

\section{Materials and Methods}

\section{Subjects}

In a single-center prospective cohort study (Trial register: NTR4764), patients with a chest-physician-confirmed COPD were recruited at the Medical Centre Leeuwarden (MCL), The Netherlands. The severity of disease was classified according to the Global Initiative for Chronic Obstructive Lung Disease (GOLD) stages [9]. All patients with COPD who were referred for CPET for the rehabilitation, dyspnea analysis, and $\mathrm{VO}_{2 \max }$ measurement, were included. Patients were excluded, if they were not able to perform CPET or MPT due to, e.g., other comorbidities. All participants gave informed consent prior to entering the study. In the clinical study, a retrospective study was performed with data of COPD patients who were analyzed because of dyspnea complaints at the Radboud University Medical Center, Nijmegen, The Netherlands. Both studies were conducted according to the Declaration of Helsinki. The prospective study (file No. NL50155.099.14/RTPO924) was approved by the Research Ethics Committee "Regionale Toetsingscommissie Patiëntgebonden Onderzoek" (RTPO). The clinical study was approved by the Research Ethics Committee of the Radboud University Nijmegen Medical Centre (file No. CMO: 2018-4357).

The severity of disease was classified according to the GOLD stages [9]. For both studies, clinically stable patients were included.

\section{Study Design}

All subjects underwent spirometry, CPET, and MPT. Spirometry was performed according to the American Thoracic Society/ European Respiratory Society guidelines for lung function measurements [10]. In both studies, MPT and CPET were performed under medication. In the prospective study, all tests were performed on one day. In the clinical study, MPT was not performed on the same day as CPET (with a maximal time span between MPT and CPET of 3 months).

\section{CPET}

All patients performed a symptom-limited incremental exercise test using an electrically braked cycle ergometer (Lode B.V.; Lode Excalibur Sport, Groningen, The Netherlands). They wore a leakage-free face mask with a turbine flow transducer and a gas sampling tube (MCL: ZAN, zan100; Accuramed, Belgium; Radboud University Medical Center: VitalAire Nederland BV, Eindhoven, The Netherlands). Measurements were performed according to the American Thoracic Society/European Respiratory Society guidelines for CPET (2003) [11]. Reference equations for calculation of predicted values were those produced by Wasserman [12].

$\mathrm{DH}$ was determined by measuring changes in the IC. First, patients were asked to sit upright and relaxed on the bike. Patients should have shown at least four consistent end-expiratory levels before carrying out an IC maneuver. The mean of three acceptable measurements $(<5 \%$ or $<100-\mathrm{ml}$ difference) was taken as baseline IC (ICrestCPET) [10]. At the moment of maximal exercise, one IC was measured (ICCPET).

\section{MPT}

Patients were seated, breathing through a mouthpiece connected to the spirometer (Masterlab PFT; Vitalaire, Germany). After a quiet and stable breathing was attained, the mean of three accept- 
Table 1. Demographics of all subjects for both the prospective and clinical study

\begin{tabular}{lcc}
\hline & $\begin{array}{l}\text { Prospective study } \\
(n=107)\end{array}$ & $\begin{array}{l}\text { Clinical study } \\
(n=48)\end{array}$ \\
\hline Age, years & $63.3(10.6)$ & $61.4(8.1)$ \\
Sex, males/females & $66 / 41(62$ vs. 38\%) & $21 / 27(44$ vs. 56\%) \\
BMI & $26.1(5.2)$ & $25.3(5.6)$ \\
FEV1, L & $1.58(0.68)$ & $1.45(0.46)$ \\
FEV1, \%pred & $53(18)$ & $50(14)$ \\
FEV1/FVC\% & $49(15)$ & $43(11)$ \\
COPD Gold I/II/III/IV & $9 / 51 / 33 / 14$ & $1 / 20 / 26 / 1$ \\
\hline
\end{tabular}

Values are presented as mean (standard deviation), unless otherwise indicated.
Table 2. Number of patients per quadrant (prospective study)

\begin{tabular}{llrc}
\hline & CPET + & CPET - & Total \\
\hline MPT + & 73 & 14 & 87 \\
MPT - & 13 & 7 & 20 \\
\hline Total & 86 & 21 & 107 \\
\hline
\end{tabular}

able maneuvers $(<10 \%$ difference) was used to establish ICrestMPT. Thereafter, patients were asked to breathe twice their resting breathing rate using a web-based metronome, with equal inspiratory and expiratory time. They were instructed to maintain their tidal volume the same as at rest. After breathing $20 \mathrm{~s}$, an IC maneuver was performed [1]. The MPT following an IC maneuver was repeated three times. A few minutes were taken in between, and the next maneuver was performed after recovery to a stable resting lung volume. The mean of three acceptable IC maneuvers was IC after MPT (ICMPT).

\section{Statistical Analysis}

Based on the results of Lahaije et al. [8], a power analysis for the prospective study was performed (5,000 Monte Carlo samples of null and alternative distribution). A difference of $10 \%$ between the $\triangle \mathrm{IC}$ of both tests was accepted (based on the repeatability coefficient found by Lahaije et al. [8]). With a power of $80 \%$, a significance of 0.05 (two-paired $t$ test), and a correlation of 0.20 (conservative approach), we calculated a sample size of 110 patients.

Baseline characteristics are described by mean \pm standard deviation or median \pm interquartile range, depending on normality. $\mathrm{DH}(\triangle \mathrm{IC})$ is the difference in \% between IC at the start (ICrestCPET, ICrestMPT) and end of the test (ICCPET, ICMPT) divided by ICrest (ICrestCPET, ICrestMPT). A more negative value of $\triangle \mathrm{IC}$ corresponds with a higher amount of induced DH. $\triangle \mathrm{IC}$ measured with CPET as reference and MPT was compared with the Bland-Altman analysis. As cutoff point for $\mathrm{DH}$, the cutoff values from the study by Lahaije et al. [8] were used. A subject

Metronome-Paced Tachypnea Test, Ready For Clinic?
Table 3. Number of patients per quadrant (clinical study)

\begin{tabular}{lccc}
\hline & CPET + & CPET - & Total \\
\hline MPT + & 29 & 10 & 39 \\
MPT- & 5 & 4 & 9 \\
\hline Total & 34 & 14 & 48 \\
\hline
\end{tabular}

was identified as a hyperinflator, if $\triangle \mathrm{IC}$ (\% of ICrest) was below -10.2 and $-11.1 \%$ in CPET and MPT, respectively. With these values, the sensitivity and specificity of MPT relative to the reference CPET were calculated.

\section{Results}

Hundred-seven clinically stable patients with COPD (FEV1\%pred $53 \pm 18$ ) were included in the prospective study between August 2014 and August 2016.

In the clinical study, forty-eight clinically stable patients with COPD (FEV1\%pred $53 \pm 17$ ) were included between October 2014 and April 2018. Both groups consisted of mild-to-very-severe COPD patients (GOLD guidelines [13]). The baseline characteristics are presented as mean (standard deviation) in Table 1.

\section{Accuracy}

The sensitivity and specificity of the MPT relative to CPET was 85 and 33\%, respectively. The positive predictive value of the MPT was $84 \%$, and the negative predictive value was $35 \%$. In the clinical study, the sensitivity and specificity of the MPT relative to CPET were 85 and $27 \%$, respectively. The positive predictive value of the MPT was $74 \%$, and the negative predictive value was $44 \%$. 


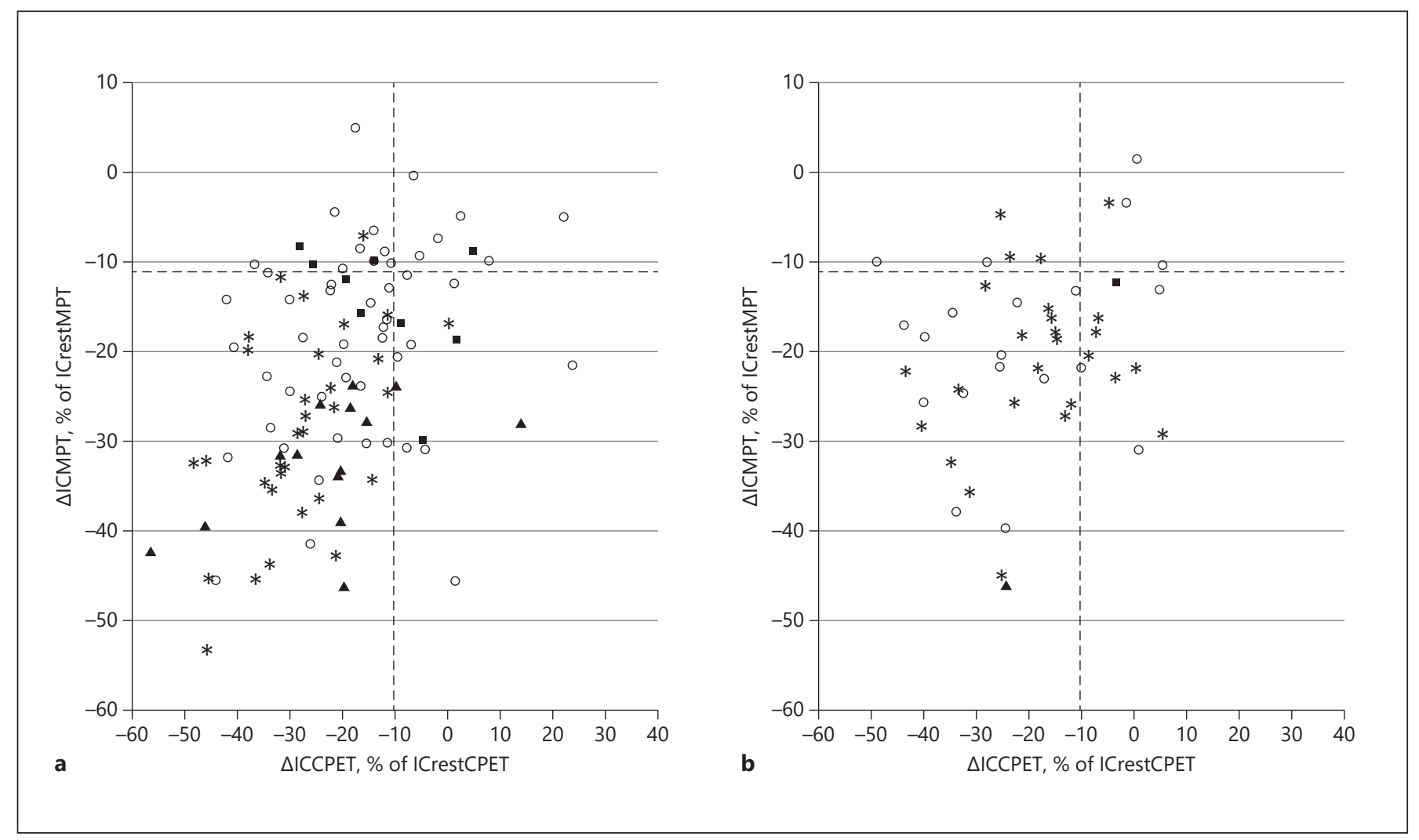

Fig. 1. Subjects correctly and incorrectly identified by MPT. On the $\mathrm{x}$-axis, $\mathrm{DH}$ was measured with CPET, and on the y-axis, DH was measured with MPT. The lower left quadrant contains the true positives, the upper right quadrant the true negatives. The upper left quadrant shows false negatives, and the lower right quadrant

shows false positives. The cutoff value was $-10.2 \%$ for CPET and $-11.1 \%$ for MPT in a the prospective study and $\mathbf{b}$ the clinical study. GOLD classification is presented with the following markers: GOLD I = filled square, GOLD II = circle, GOLD III = asterisk, GOLD IV = filled triangle.

See Figure 1a (prospective), $1 \mathrm{~b}$ (clinical) for quadrant figures, and Tables 2 and 3 for the number of patients per quadrant.

\section{Comparison DH with CPET and MPT}

In the prospective study, the mean $\triangle \mathrm{ICMPT}$ (\% of ICrestMPT) was $-23 \pm 12 \%(-0.57 \pm 0.36 \mathrm{~L})$, and the mean $\triangle \mathrm{ICCPET}$ ( $\%$ of ICrestCPET) was $-21 \pm 15 \%(-0.51$ $\pm 0.41 \mathrm{~L}$ ). In the clinical study, the mean $\triangle \mathrm{ICMPT}$ (\% of ICrestMPT) was $-19 \pm 10 \%(-0.41 \pm 0.23 \mathrm{~L})$, and the mean $\triangle \mathrm{ICCPET}$ (\% of ICrestCPET) was $-19 \pm 15.0 \%$ $(-0.49 \pm 0.42 \mathrm{~L})$. There is no significant difference in the mean $\triangle \mathrm{ICCPET}$ (\% of ICrestCPET) and $\triangle \mathrm{ICMPT}$ (\% of ICrestMPT) between both studies.

In both the prospective and clinical study, there was no significant difference between the mean $\triangle \mathrm{ICCPET}$ (\% of ICrestCPET) and $\triangle \mathrm{ICMPT}$ ( $\%$ of ICrestMPT) $(p=0.07$ and 0.73 , respectively). In Figure $2 \mathrm{a}$ and $\mathrm{b}$, the Bland-Alt- man analysis of both studies can be found. The bias between $\triangle \mathrm{ICCPET}$ and $\triangle \mathrm{ICMPT}$ was 2.6 and $0.8 \%$, respectively.

\section{Discussion}

In this study, the focus was to investigate the diagnostic accuracy of MPT to detect DH in two settings. First, a prospective study was performed, and second, a retrospective database study was performed at the Radboud University Medical Center. The sensitivity of the MPT was good; in both studies, $85 \%$ of the subjects were correctly identified as hyperinflators. However, the specificity was low. The mean difference between $\triangle \mathrm{IC}$ (\% of ICrest), measured with both methods, was small, and in both studies, the limits of agreement were wide. 


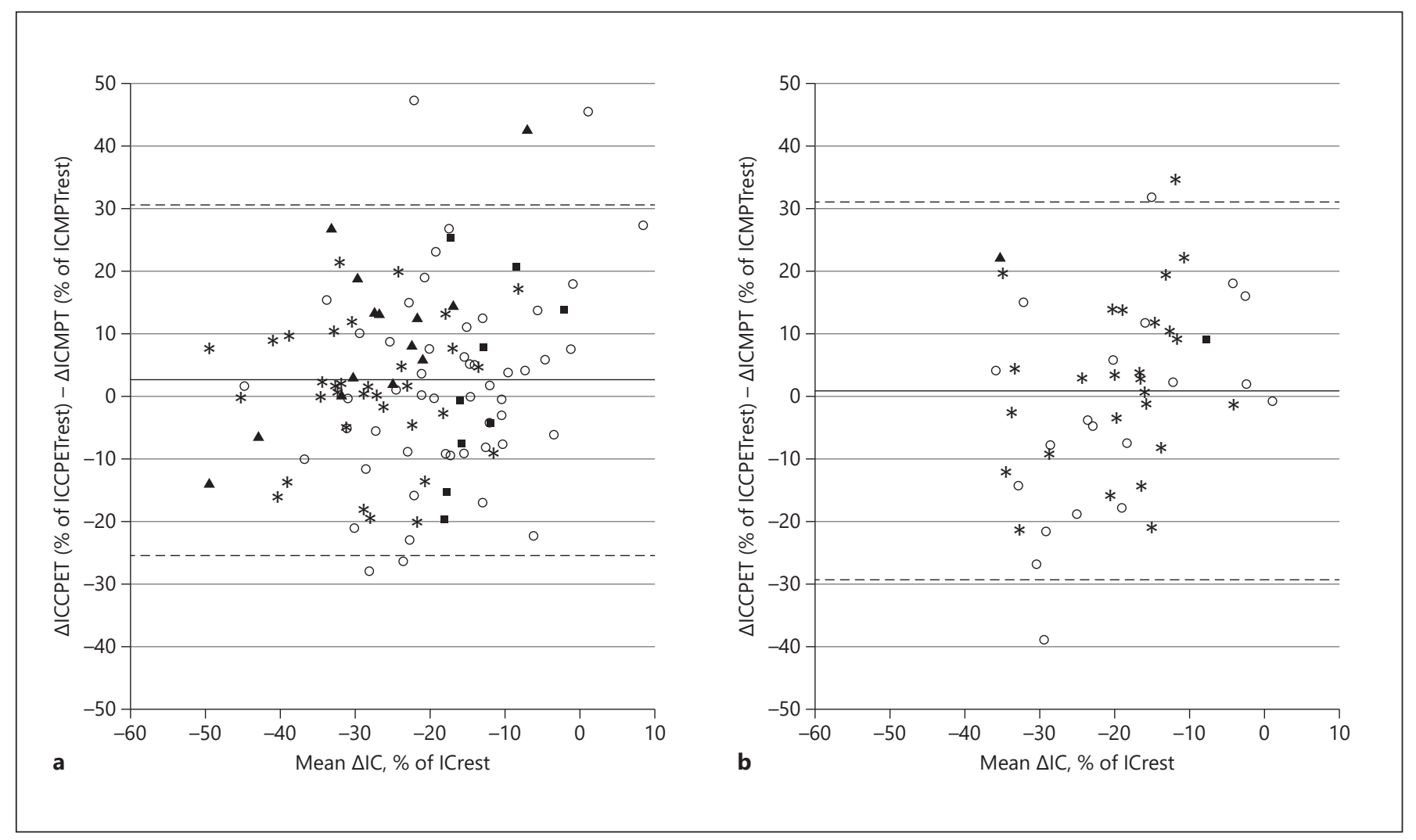

Fig. 2. Bland-Altman plot comparing the relative CPET-induced $\mathrm{DH}$ with MPT-induced $\mathrm{DH}$. A positive value on the $y$-axis means an overestimation of DH as measured with MPT. GOLD classification is presented with the following markers: GOLD $\mathrm{A}=$ filled square,

GOLD B = circle, GOLD C = asterisk, GOLD D = filled triangle. The bias (B) is presented as the solid line; limits of agreement (L) are dashed lines. a Prospective study: $\mathrm{B}=+2.6 \% ; \mathrm{L}=30.6 \%$, and $-25.6 \%$. b Clinical study: $\mathrm{B}=+0.8 \%, \mathrm{~L}=31.0$, and $-29.1 \%$.

\section{Accuracy}

In both the prospective and clinical study, the sensitivity was $85 \%$. The specificity was 33 and $27 \%$, respectively. From these results, it can be concluded that the overall accuracy of MPT is questionable. Especially, the specificity of MPT is low, which means that MPT fails to correctly identify nonhyperinflators. In practice, this could lead to overdiagnosis of $\mathrm{DH}$, if MPT would replace CPET. However, the sensitivity of the MPT is good, which means that the chance is high that a subject who shows DH during MPT has DH during CPET.

In the study by Lahaije et al. [8], a sensitivity and specificity were found of 85 and $85 \%$, respectively. Calligaro et al. [3] found a sensitivity and specificity of 90 and $38 \%$, respectively. The sensitivity and specificity in our study and in the study by Calligaro et al. [3] are comparable. The difference in sensitivity and specific- ity in the studies by Lahaije et al. [8] and our study might be explained by a difference in population. Especially for CPET-induced $\mathrm{DH}$, a different population can have a consequence. In the study by Lahaije et al. [8], the thirty-five COPD patients were included at their initial screening for pulmonary rehabilitation. They concluded that there might have been a selection bias because a higher prevalence of DH (in CPET) may be expected in patients with COPD referred for rehabilitation. During MPT, the chance of developing $\mathrm{DH}$ is high, even in healthy subjects $[8,14]$. While in CPET, the chance of inducing DH is smaller. In our study, the population with milder forms of COPD could have introduced more false-positives (i.e., $\mathrm{DH}$ in MPT+ and DH in CPET-). Moreover, we did not include healthy subjects, which was also the case in the study by Calligaro et al. [3]. This makes the true-negative group smaller, affecting the specificity. This can be supported 
by the findings in the study by Lahaije et al. [8], in which a lower specificity (71\%) was found in a subanalysis excluding healthy subjects.

\section{Comparison $\mathrm{DH}$}

In the prospective and clinical study, the bias was +2.6 and $+0.8 \%$, a small overestimation of DH with MPT. Limits of agreement were wide: 30.6 and $-25.6 \%$, and 31.0 and $-29.1 \%$, respectively. Lahaije et al. [8] found an overestimation of MPT of $3.0 \%$ on CPET, with limits of agreement of $\pm 24 \%$. Calligaro et al. [3] state that MPT overestimates $\mathrm{DH}$; the corresponding bias is not given. All studies show that MPT tends to slightly overestimate $\mathrm{DH}$, which is neglectable from a clinical point of view.

One explanation is that MPT- and CPET-induced DH are physiologically different. In both the clinical and prospective study, all subjects had to breathe at twice their resting frequency during MPT. In the CPET, the respiratory frequency is not imposed. Most of the time, the maximal respiratory rate in CPET is smaller than in MPT and often not equal at the end of the test. In addition, during MPT, subjects were encouraged to keep the tidal volume equal to the resting tidal volume, while this was not the case in CPET. In the study by Cooper et al. [14], it is demonstrated that not only breathing frequency determines the amount of $\mathrm{DH}$. The ratio between inspiratory and expiratory time is also an important factor. Moreover, it can be hypothesized that tidal volume also determines the induction of DH. Thus, it can be reasoned that $\mathrm{DH}$ induced by MPT and CPET are physiologically different and not comparable. Moreover, it should be kept in mind that the MPT has a "subject-specific" protocol (i.e., subject 1 has a MPT frequency of 40 , while subject 2 has a MPT frequency of 20). This could mean that DH measured with MPT is not comparable between subjects.

In addition to the physiological difference of the underlying mechanism, a difference in the measurement setup could further increase the difference in the induced $\mathrm{DH}$. In CPET, the subject wears a mask while the subject breathes through a mouth piece during MPT. During CPET, a subject can use the pursed-lip breathing technique, which could help the patient prevent the induction of $\mathrm{DH}$, or at least reduce the amount of $\mathrm{DH}$.

\section{Clinical Implications}

Our studies show a nonsignificant difference between the mean $\triangle \mathrm{ICCPET}$ and $\triangle \mathrm{ICMPT}$. Therefore, in a group, the mean DH measured with MPT and CPET is interchangeable. Together with the studies of Lahaije et al. [8] and Calligaro et al. [3], it can be concluded that studies in- vestigating the effect of therapy on $\mathrm{DH}[2,3]$, the coherence of DH with other parameters [15], or the development of $\mathrm{DH}$ over time in a group with COPD patients $[1,7]$ can be simplified by measuring with MPT instead of using the more labor-intensive CPET. On an individual level, MPT should be used cautiously to determine, if a subject is a (non-) hyperinflator. It should be kept in mind that this leads to false-positives (i.e., labelled as a hyperinflator, while he/she is not a hyperinflator). In addition, the amount of $\mathrm{DH}(\triangle \mathrm{IC}$ [\% of ICrest]) is not comparable between the two methods for an individual, as the Bland-Altman analysis showed that the difference between $\triangle \mathrm{ICCPET}$ (\% of ICrest) and $\triangle \mathrm{ICMPT}$ (\% of ICrest) varies greatly. $\mathrm{DH}$ is probably hard to express in numbers and is dependent on activity/test, so it should be described as present or absent.

\section{Limitations}

There remains uncertainty on the validity of the measurement of DH with the reference method CPET. CPET is a time-consuming test, and effort is needed from both the patient and physician. The instructions might be harder to follow during CPET than MPT. However, it is beyond the scope of this article to elaborate further on the limitations of CPET.

In the clinical study, the tests were not necessarily carried out on the same day. The results of the study by Hannink et al. [7] suggest that DH changes over time in patients with COPD. Therefore, DH measured with CPET and MPT can be different. This would suggest that the reliability of the outcomes is less accurate than when CPET and MPT measurements take place on the same day. However, it is reasoned that the effect of measurement of $\mathrm{DH}$ on different days is neglectable. Indeed, there was no difference in sensitivity (85\%) between both the prospective and clinical study, and the difference in specificity (33 and $27 \%$ ) is minimal.

Another limitation might be that we did not use the optimal cutoff value to determine the accuracy of MPT. For example, another cutoff value is used in the study by Calligaro et al. [3]. DH was considered present, if the difference in IC was more than 1.96 SD below ICrest. We used the cutoff values of Lahaije et al. [8] to prevent even more cutoff values to appear in the literature. The cutoff values were 10.2 and $11.1 \%$ for the CPET and MPT test, respectively, which were based on ROC analysis. It is hypothesized that this value is close to the true optimal cutoff value, as these values were close to the repeatability coefficient of the IC in rest.

In conclusion, MPT seems to be a good replacement for CPET in group studies. The mean amount of $\mathrm{DH}$ on group 
basis does not differ between CPET and MPT. On an individual level and for diagnostic use, MPT should be used with caution to identify hyperinflators; it should be kept in mind that MPT overdiagnoses $\mathrm{DH}$. The amount of DH should not be interchanged between CPET and MPT. To increase the clinical value of the MPT, future studies should focus on finding the clinical accuracy of the MPT in an individual based on pre- and post-treatment measurements (e.g., rehabilitation or medical intervention). Moreover, more research on the standardization of the MPT protocol (e.g., tidal volume, breathing frequency) could be important to increase its clinical value.

\section{Acknowledgements}

At the Radboud University Medical Center, financial aid was provided by an educational grant of Chiesi Pharmaceuticals B.V., The Netherlands.

\section{Statement of Ethics}

Subjects (or their parents or guardians) have given their written informed consent. The study protocol has been approved by the research institute's committee on human research.

\section{Disclosure Statement}

D.M., E.V., H.-K., J.M., and A.H. have no conflicts of interest to declare. H.H. reports an educational grant from Chiesi, the Netherlands, during the conduct of the study.

\section{Funding Sources}

The authors received an unrestricted educational grant from Chiesi, The Netherlands.

\section{Authors Contributions}

D.M. takes the responsibility for the content of the paper, including the data and analysis; she is the guarantor. D.M. and E.V. made substantial contributions to the analysis of the data and their interpretation. E.V., A.H.-K., J.M., A.H., and H.H., made substantial contributions to the conception and design. E.V. and A.H.-K. made a substantial contribution to data acquisition. D.M., E.V., A.H.-K., J.M., A.H., and H.H. drafted or revised the article critically, provided final approval of the version to be published, and agreed to be accountable for all aspects of the work.

\section{References}

1 Gelb AF, Gutierrez CA, Weisman IM, Newsom R, Taylor CF, Zamel N. Simplified detection of dynamic hyperinflation. Chest. 2004 Dec;126(6):1855-60.

2 Fujimoto K, Yoshiike F, Yasuo M, Kitaguchi $\mathrm{Y}$, Urushihata K, Kubo K, et al. Effects of bronchodilators on dynamic hyperinflation following hyperventilation in patients with COPD. Respirology. 2007 Jan;12(1):93-9.

3 Calligaro GL, Raine RI, Bateman ME, Bateman ED, Cooper CB. Comparing dynamic hyperinflation and associated dyspnea induced by metronome-paced tachypnea versus incremental exercise. COPD. 2014 Feb;11(1):105-12.

4 Fujimoto K, Yamazaki H, Ura M, Kitaguchi Y. Efficacy of tiotropium and indacaterol monotherapy and their combination on dynamic lung hyperinflation in COPD: a random openlabel crossover study. Int J Chron Obstruct Pulmon Dis. 2017 Nov;12:3195-201.

5 van Geffen WH, Kerstjens HA. Static and dynamic hyperinflation during severe acute exacerbations of chronic obstructive pulmonary disease. Int J Chron Obstruct Pulmon Dis. 2018 Apr;13:1269-77.
6 Klooster K, ten Hacken NH, Hartman JE, Sciurba FC, Kerstjens HA, Slebos DJ. Determining the Role of Dynamic Hyperinflation in $\mathrm{Pa}$ tients with Severe Chronic Obstructive Pulmonary Disease. Respiration. 2015;90(4):306-13.

7 Hannink J, Lahaije A, Bischoff E, van Helvoort $\mathrm{H}$, Dekhuijzen R, Schermer T, et al. Dynamic hyperinflation after metronome-paced hyperventilation in COPD - a 2 year followup. Respir Med. 2010 Nov;104(11):1700-5.

8 Lahaije AJ, Willems LM, van Hees HW, Dekhuijzen PN, van Helvoort HA, Heijdra YF. Diagnostic accuracy of metronome-paced tachypnea to detect dynamic hyperinflation. Clin Physiol Funct Imaging. 2013 Jan;33(1):62-9.

9 Rabe KF, Hurd S, Anzueto A, Barnes PJ, Buist SA, Calverley P, et al.; Global Initiative for Chronic Obstructive Lung Disease. Global strategy for the diagnosis, management, and prevention of chronic obstructive pulmonary disease: GOLD executive summary. Am J Respir Crit Care Med. 2007 Sep;176(6):532-55.

10 Miller MR, Hankinson J, Brusasco V, Burgos F, Casaburi R, Coates A, et al.; ATS/ERS Task Force. Standardisation of spirometry. Eur Respir J. 2005 Aug;26(2):319-38.

11 American Thoracic Society; American College of Chest Physicians. ATS/ACCP Statement on Cardiopulmonary Exercise Testing. Am J Respir Crit Care Med. 2003 Jan;167(2):211-77.
12 Wasserman K, Hansen JE, Sue DY. Principles of exercise testing and interpretation: including pathophysiology and clinical applications. 5th ed. Philadelphia: Lippincott, Williams \& Wilkins; 2005. p. 4

13 Vogelmeier CF, Criner GJ, Martinez FJ, Anzueto A, Barnes PJ, Bourbeau J, et al.; Global Strategy for the Diagnosis, Management, and Prevention of Chronic Obstructive Lung Disease 2017 Report. Global Strategy for the Diagnosis, Management, and Prevention of Chronic Obstructive Lung Disease 2017 Report. GOLD Executive Summary. Am J Respir Crit Care Med. 2017 Mar;195(5):557-82.

14 Cooper CB, Calligaro GL, Quinn MM, Eshaghian P, Coskun F, Abrazado M, et al. Determinants of dynamic hyperinflation during metronome-paced tachypnea in COPD and normal subjects. Respir Physiol Neurobiol. 2014 Jan;190:76-80.

15 Kitaguchi Y, Fujimoto K, Hanaoka M, Honda T, Hotta J, Hirayama J. Pulmonary function impairment in patients with combined pulmonary fibrosis and emphysema with and without airflow obstruction. Int $\mathrm{J}$ Chron $\mathrm{Ob}$ struct Pulmon Dis. 2014 Jul;9:805-11.
Metronome-Paced Tachypnea Test, Ready For Clinic? 